\title{
Stationary bottom generated velocity fluctuations in one-dimensional open channel flow
}

\author{
Bartele de Jong \\ Department of Applied Mathematics, University of Twente, P.O. Box 217, 7500 AE Enschede, Netherlands
}

(Received 12 May 1992; revised 28 December 1992; accepted 30 December 1992)

\begin{abstract}
Statistical characteristics are calculated for stationary velocity fluctuations in a one-dimensional open channel flow with a given vertical velocity profile and with one-dimensional irregular bottom waves, characterized by a spectral density function. The calculations are based on an approximate calculation of the velocity fluctuations in the fluid generated by a harmonic corrugation of the bottom. As linearized dynamical equations are used, the velocity fluctuations caused by random bottom disturbances may be obtained by superposition. The dynamics of the motion is assumed to be governed by the Orr-Sommerfeld equation representing the internal wave motion in the fluid. This equation is solved in an approximate manner by reducing it in the upper layer of the fluid to the Rayleigh equation. Close to the bottom we simplify it to a shape still containing the essentials of the viscous behaviour of the flow. Numerical examples and a tentative qualitative comparison with experimental data are given.
\end{abstract}

\section{INTRODUCTION}

Morphological and ecological processes in natural water bodies such as estuaries, rivers or seas are largely determined by random turbulent fluctuations of the velocity about its equilibrium value. These fluctuations influence, for example, the diffusion of momentum and the diffusion of material, and are responsible for the bed patterns at the bottom of alluvial channels, which in some cases may be rather regular. This regularity suggests that at least in certain circumstances these velocity fluctuations are based on a well-defined physical mechanism.

In this paper one-dimensional channel flows are considered, such as are observed approximately in rivers, estuaries or in coastal seas as a result of tidal influence. In this case, turbulence is mainly generated by the bed and the free surface, owing to the irregularities at the bottom and the free surface. For this reason we may expect that the statistical properties of the irregular turbulent flow motion and the related transport phenomena are 
determined largely by the statistical properties of the bed elevations and the free surface waves.

As turbulence has a very complex structure, with phenomena such as bursts of shear stress or vortex shedding, it is very difficult to describe. For that reason, turbulent flow models are still rather heuristic. Many of these models are based on an appropriate definition of the eddy viscosity. Also, experimental input is often needed to obtain tractable equations for the turbulent quantities (see, e.g. Rodi, 1980). In this paper we will concentrate only on the regular wavy part of the turbulence which does not cover phenomena such as bursting or breaking of waves. We further restrict our discussion to stationary two-dimensional waves as generated by one-dimensional bottom waves. These waves are described using the phenomenon of internal waves as observed in a stratified flow (Turner, 1973; Lighthill, 1978). It is clear that fully developed turbulence, which is essentially three dimensional, cannot be described by this simple two-dimensional wave model. However, in the literature we find several examples where the importance of these waves is evident. By comparing calculations with experiments, De Jong (1989) showed that in a channel flow with an erodible bed the generation of the initially regular dune and ripple pattern on a flat bed is due to stationary internal waves corresponding to the eigenoscillations of the flow. Further, Kranenburg et al. (1991) showed, by observations in the Rotterdam Waterway, that in field situations also a regular bottom pattern corresponds to resonant wave phenomena in the flow.

In this paper we assume a one-dimensional channel flow with a given vertical velocity distribution but with a uniform density. The bottom is one-dimensionally randomly corrugated with a given spectral density distribution. The free surface is supposed not to be disturbed by wind or other external influences. The Orr-Sommerfeld (OS) equation is used to relate a harmonic corrugation at the bottom to the generated harmonic velocity fluctuations in the fluid. As this equation is linear, the disturbances in the fluid caused by random disturbances at the bottom are obtained by a simple superposition. The OS equation will be solved in an approximate manner. We assume the flow in the upper layer to be governed by the Rayleigh equation. Close to the bottom a more accurate solution including viscosity is needed, as we have to relate the velocity fluctuations in the fluid to the bottom irregularities by imposing suitable boundary conditions. In this paper, this is achieved by determining the four elementary solutions of the fourth-order OS equation in a heuristic manner by reducing this equation to appropriate forms still containing the essential properties of the flow. The OS equation has been applied in many earlier papers to describe oscillations in turbulent flow; e.g. Landahl (1967) used this equa- 
tion to model plane channel flow by travelling damped wave oscillations. The excitation is there provided by non-linear fluctuating stress terms.

In Section 2 we formulate the general boundary value problem governing the internal waves generated by a one-dimensional harmonic bed corrugation. In Section 3 the heuristic solution of this problem is described. In Section 4 numerical results are calculated for statistical quantities characterizing the fluctuations for various flow and bed parameters. The final section presents a summary and discussion.

\section{MATHEMATICAL FORMULATION}

We assume the open channel flow to extend from $x=-\infty$ to $x=+\infty$, with the $x$-axis in the longitudinal direction and coinciding with the mean level of the bed. The $z$-axis is vertical and positive in the upward direction. At the bottom of the channel we assume one-dimensional random elevations which vary only in the $x$-direction.

The basic equations governing the wave propagation through a viscous flow are the Navier-Stokes equation (Turner, 1973)

$$
\frac{\mathrm{D} \boldsymbol{u}}{\mathrm{D} t}=\frac{\partial \boldsymbol{u}}{\partial t}+(\boldsymbol{u} \cdot \nabla) \boldsymbol{u}=-\nabla\left(\frac{p}{\rho}\right)+\boldsymbol{g}+\nu \nabla^{2} \boldsymbol{u}
$$

and the continuity equation

$$
\nabla \cdot \boldsymbol{u}=0
$$

where $\mathrm{D} / \mathrm{D} t$ denotes differentiation following the motion, $u=(U+u, w)$ represents the velocity vector with mean value $U=U(z)$ in the $x$-direction and disturbances $u$ and $w, \rho$ is the constant density, $g=(0,-g)$ is the acceleration due to gravity, $p=p_{0}+p_{e}$ is the pressure, which is the sum of the undisturbed value $p_{0}$ and the disturbance $p_{e}$, and $\nu$ represents the kinematic viscosity of the fluid. In the usual way we satisfy Eqn. (2) by writing $u$ and $w$ in terms of the disturbed streamfunction $\psi$ :

$u=\frac{\partial \psi}{\partial z}, \quad w=-\frac{\partial \psi}{\partial x}$

Next, we eliminate $p$ from Eqn. (1), linearize with respect to the disturbances and write $\psi$ as a harmonic function in $x$, representing stationary waves

$\psi=Q \mathrm{e}^{i k x}$

with $k$ the wavenumber and $Q$ a depth-dependent function. The final 
result is the well-known OS equation for the special case of stationary waves (Drazin and Reid, 1982):

$U\left(\frac{\mathrm{d}^{2} Q}{\mathrm{~d} z^{2}}-k^{2} Q\right)-\frac{\mathrm{d}^{2} U}{\mathrm{~d} z^{2}} Q=-\frac{i}{k \operatorname{Re}}\left(\frac{\mathrm{d}^{4} Q}{\mathrm{~d} z^{4}}-2 k^{2} \frac{\mathrm{d}^{2} Q}{\mathrm{~d} z^{2}}+k^{4} Q\right)$

In this equation all quantities have been made dimensionless, with the depth $d$ and the mean velocity $\bar{U}$ in the channel leading to the introduction of the Reynolds number $\operatorname{Re}=\bar{U} d / \nu$.

As boundary conditions we have first the condition of zero velocity at the bottom which, by using Eqns. (3) and (4), may be expressed in the form

$Q=0, \quad \frac{\mathrm{d} Q}{\mathrm{~d} z}=0, \quad z=0$

At the free surface $\psi$ has to satisfy the kinematic condition, which in linearized form has the shape (Stoker, 1957; De Jong, 1989)

$\frac{\mathrm{d} \eta}{\mathrm{d} x}=\frac{w}{U+u}, \quad z=1$

and the dynamic equation

$U \frac{\partial \psi}{\partial z}+g \eta=0, \quad z=1$

where $\eta=\eta(x)$ is a small elevation at the free surface. In deriving Eqn. (8) we assumed the mean velocity to be homogeneous at the free surface, which is, in general, approximately the case. Using Eqns. (3), (7) and (8), we obtain the linearized relation

$-g \frac{\partial \psi}{\partial x}+U^{2} \frac{\partial^{2} \psi}{\partial x} \frac{\partial z}{\partial z}=0, \quad z=1$

By substituting Eqn. (4) in Eqn. (9) we derive the free surface condition, which we write in dimensionless form

$U^{2} Q^{\prime}-\mathrm{Fr}^{-2} Q=0, \quad z=1$

where $\mathrm{Fr}=\bar{U} /(\mathrm{gd})^{1 / 2}$ represents the Froude number and $Q^{\prime}=\mathrm{d} Q / \mathrm{d} z$.

The fourth boundary condition refers to the bed wave elevations $\zeta$, which are assumed to be normally distributed with given spectral density function $S_{\zeta \zeta}(k)$ defined for the range $0 \leqslant k<\infty$. In engineering practice, these stationary random waves are often expressed by the following stochastic integral representation (Pierson et al., 1958; Ochi, 1982):

$\zeta(x, t)=\int_{0}^{\infty} \cos [k x+\epsilon(k)]\left[2 S_{\zeta \zeta}(k) \mathrm{d} k\right]^{1 / 2}$ 
which should be interpreted as

$\zeta(x, t)=\sum_{r=0}^{N} \cos \left(k_{2 r+1} x+\epsilon_{2 r+1}\right)\left[2 S_{\zeta \zeta}\left(k_{2 r+1}\right) \Delta k\right]^{1 / 2}, \quad z=1$

with the limits $k_{2 N+1} \rightarrow \infty$ and $\Delta k=k_{2 r+2}-k_{2 r} \rightarrow 0$ for $k_{0}=0$ and $k_{0}<$ $k_{1}<\ldots<k_{2 N+1}$. The phase angles $\epsilon_{2 r+1}$ are mutually independent random variables distributed uniformly over the range $(0,2 \pi)$. As Eqn. (5) is linear, we may restrict our discussion to a single harmonic component

$\zeta=\left[2 S_{\zeta \zeta}(k)\right]^{1 / 2} \cos (k x+\epsilon)$

The effect of the complete wave system is obtained by superposition. It is observed that Eqn. (11) leads to the familiar expression $\overline{\zeta^{2}}=\int_{0}^{\infty} S_{\zeta \zeta}(k) \mathrm{d} k$ for the mean energy per unit area, which highlights the convention in evaluating mean square values of expressions involving integral expressions of the form of Eqn. (11). The condition relating the function $Q$ defined in Eqn. (4) to the bottom elevation $\zeta$ is easy to derive from Eqn. (1) (Schlichting, 1968). At the bottom, where $U=u=w=0$, the equation for the horizontal component reduces to

$\nu \frac{\partial^{2}(U+u)}{\partial z^{2}}=\frac{1}{\rho} \frac{\mathrm{d} p}{\mathrm{~d} x}, \quad z=0$

For the pressure $p$ at the bed surface we take the simple hydrostatic pressure and neglect the free surface disturbance caused by the bed waves. In view of the discussion of Raudkivi (1990), this also is a rather rough approximation. When the bottom is harmonically corrugated with amplitude $\zeta_{a}, \zeta=\zeta_{a} \mathrm{e}^{i k x}$, we obtain

$p=p_{0}+\rho g\left(\bar{\zeta}-\zeta_{a} \mathrm{e}^{i k x}\right)$

in which $p_{0}$ is the constant atmospheric pressure and $\bar{\zeta}$ the mean level of the bottom with respect to the mean water level. Substituting Eqn. (15) into (14) leads in dimensionless form to the following relation:

$\frac{\partial^{2}(U+u)}{\partial z^{2}}=\operatorname{Re} \operatorname{Fr}^{-2}\left(\frac{\mathrm{d} \bar{\zeta}}{\mathrm{d} x}-i \zeta_{a} k \mathrm{e}^{i k x}\right)$

As the mean velocity satisfies the familiar expression $\mathrm{d}^{2} U / d z^{2}=$ $\operatorname{Re} \operatorname{Fr}^{-2} \mathrm{~d} \bar{\zeta} / \mathrm{d} x$, we have for the disturbed velocity $u$ the relation

$\frac{\partial^{2} u}{\partial z^{2}}=-i \operatorname{Re} \operatorname{Fr}^{-2} \zeta_{a} k \mathrm{e}^{i k x}$ 
Combining Eqns. (3), (4) and (17), we find

$$
\frac{\mathrm{d}^{3} Q}{\mathrm{~d} z^{3}}=-i \operatorname{Fr}^{-2} \operatorname{Re} k \zeta_{a}, \quad z=0
$$

Because, according to Eqn. (13), $\zeta_{a}=\left[2 S_{\zeta \zeta}(k)\right]^{1 / 2}$, the amplitude or modulus of $\mathrm{d}^{3} Q / \mathrm{d} z^{3}$ satisfies the relation

$$
\left|\frac{\mathrm{d}^{3} Q}{\mathrm{~d} z^{3}}\right|=\operatorname{Fr}^{-2} \operatorname{Re} k\left[2 S_{\zeta \zeta}(k)\right]^{1 / 2}, \quad z=0
$$

Equation (5) and the conditions (6), (10) and (19) compose the boundary value problem for the amplitude $Q$ of the streamfunction as result of the harmonic corrugation of the bed with amplitude $\zeta_{a}=\left[2 S_{\zeta \zeta}(k)\right]^{1 / 2}$ and wavenumber $k$.

\section{A HEURISTIC SOLUTION METHOD}

The straightforward solution of the OS equation is still a complicated task, in particular when it is part of a more general boundary value problem as formulated in the preceding section (see, e.g. the monographs of Betchov and Criminale (1967) and Drazin and Reid (1982)). For this reason, we will use in this paper a relatively simple and rather heuristic solution method as described, for instance, by Tollmien (1947), Holstein (1950) and Drazin and Reid (1982). The heuristics of the method consist of the reduction of the OS equation to simpler forms by neglecting less relevant terms on the basis of physical considerations.

We assume that the mean velocity $U$ has everywhere the same sign. Then Eqn. (5) is singular only at the bottom, where $U=0$. This means that close to the bottom the viscous right-hand terms cannot be neglected. In the upper region of the flow, where $U$ changes only gradually we may reduce Eqn. (5) to the Rayleigh equation:

$$
\frac{\mathrm{d}^{2} Q}{\mathrm{~d} z^{2}}-\left(\frac{U^{\prime \prime}}{U}+k^{2}\right) Q=0
$$

At the free surface Eqn. (20) has to satisfy the conditions

$$
U^{2} Q^{\prime}-\mathrm{Fr}^{-2} Q=0 \text { and } Q=1 \text { for } z=1
$$

where the first condition is the free surface condition (10). The value $Q=1$ at the free surface is still arbitrary and will be replaced at the end by the normalization given by Eqn. (19). As mentioned above, Eqn. (5) has a singular point at the bottom. Viscous effects become important there and Eqn. (20) is no longer a satisfactory representation. Methods are available 
to produce, in a bounded region of such points, uniform asymptotic series solutions of Eqn. (5) (see, e.g. Drazin and Reid, 1982). However, as noted above, we will choose here a simpler approach to obtain asymptotic expressions for the solutions valid close to the bottom. This method is often indicated as the 'heuristic method' in the literature (Drazin and Reid, 1982) and is, in fact, the outcome of the first major effort to produce approximate solutions of the OS equation (Tollmien, 1929, 1947; Holstein, 1950). The main reason for choosing this approach is that not much is known about the shape of the mean velocity profile close to the bottom, in particular when the bottom has ripples and dunes, which is the case we consider. It is clear that the two linear independent solutions of Eqn. (20) should be approximations to solutions of Eqn. (5). It may be shown (Tollmien, 1947; Holstein, 1950; Reid, 1965) that one solution of Eqn. (20) may be given as a regular series expansion

$\phi_{1}(z)=z+\frac{1}{2} \frac{U_{0}^{\prime \prime}}{U_{0}^{\prime}} z^{2}$

where only the first two terms are retained. The derivatives $U_{0}^{\prime}=U^{\prime}(0)$ and $U_{0}^{\prime \prime}=U^{\prime \prime}(0)$ appear in Eqn. (22), as we used the approximations $U(z)=U_{0}^{\prime} z$ and $U^{\prime \prime}(z)=U_{0}^{\prime \prime}$. Higher-order terms of the expansion (22) will demand more information on the mean velocity. It may be shown that the other solution $\phi_{2}(z)$ of Eqn. (20) leads to a singular behaviour of the velocities close to the bottom. Apparently, a viscous correction close to the bottom is necessary to avoid this unwanted phenomenon. This is achieved by including in Eqn. (20) the viscous term - $(i / k \mathrm{Re}) Q^{\prime \prime \prime}$ of Eqn. (5) and dropping the term $k^{2} Q$, leading to

$U Q^{\prime \prime}-U^{\prime \prime} Q=-\frac{i}{k \operatorname{Re}} Q^{\prime \prime \prime}$

The solution $\phi_{2}$ is now determined as a solution of Eqn. (23) with the special form

$\phi_{2}(\eta)=1+\epsilon \frac{U_{0}^{\prime \prime}}{U_{0}^{\prime}} \tilde{\phi}_{21}(\eta)$

where $\eta=z / \epsilon$ and $\epsilon=\left(k \operatorname{Re} U_{0}^{\prime}\right)^{-1 / 3}$. By substituting Eqn. (24) in (23) we find that the function $\tilde{\phi}_{21}$ has to satisfy the equation

$\frac{\mathrm{d}^{4} \tilde{\phi}_{21}}{\mathrm{~d} \eta^{4}}-i \eta \frac{\mathrm{d}^{2} \tilde{\phi}_{21}}{\mathrm{~d} \eta^{2}}=-i$

where we made the approximations $U(z)=U_{0}^{\prime} z, U^{\prime \prime}(z)=U_{0}^{\prime \prime}$ and $Q=1$. The discussion leading to Eqns. (24) and (25) is valid for values of $\epsilon$ so 
small that $\eta$ already has large values while $z$ is still small. It is then found that the second elementary solution of Eqn. (20) has a leading term of one, as also has been prescribed in Eqn. (24), whereas for large values of $\eta$ this solution goes to infinity as $\eta \cdot \ln (\eta)$. This last property is used as the boundary condition to determine the solution $\tilde{\phi}_{21}$ of Eqn. (25). In this way, it is found that for large values of $\eta$, where viscosity is negligible, the behaviour of $\phi_{2}$ is the same as that of the second elementary solution of Eqn. (20). For further details of the analysis the reader is referred to the above-mentioned references. Holstein (1950) has tabulated the function $\mathrm{d}^{2} \tilde{\phi}_{21} / \mathrm{d} \eta^{2}$ and its integrals $\mathrm{d} \tilde{\phi}_{21} / \mathrm{d} \eta$ and $\tilde{\phi}_{21}$ for the range $\eta=0(0.5) 8$. These tabulations have also been given by Stuart (1963).

The other two approximate solutions $\phi_{3}$ and $\phi_{4}$ of Eqn. (5) are dominated by the viscous behaviour of the fluid and are approximated by solving the equation (Tollmien, 1947; Holstein, 1950)

$$
\frac{\mathrm{d}^{4} Q}{\mathrm{~d} z^{4}}-i k \operatorname{Re} U \frac{\mathrm{d}^{2} Q}{\mathrm{~d} z^{2}}=0
$$

This equation is derived from Eqn. (5) by retaining only the highest derivatives on the left- and right-hand side of Eqn. (5), as the viscous solutions are rapidly changing. Two solutions of Eqn. (26) are determined by $\mathrm{d}^{2} Q / \mathrm{d} z^{2}=0$, yielding the leading terms of the earlier determined solutions $\phi_{1}$ and $\phi_{2}$. The two other solutions of Eqn. (26) are determined by considering it as a second-order equation in $\mathrm{d}^{2} Q / \mathrm{d} z^{2}$. By setting

$$
\begin{aligned}
& U(z)=U_{0}^{\prime} z, \quad \epsilon=\left(k \operatorname{Re} U_{0}^{\prime}\right)^{-1 / 3}, \quad \eta=z / \epsilon, \quad \xi=\eta \mathrm{e}^{i \pi / 6} \\
& \chi(\eta)=\mathrm{d}^{2} Q / \mathrm{d} z^{2} \text { or } \Delta(\xi)=\mathrm{d}^{2} Q / \mathrm{d} z^{2}
\end{aligned}
$$

we may rewrite Eqn. (26) as

$$
\frac{\mathrm{d}^{2} \Delta(\xi)}{\mathrm{d} \xi^{2}}-\xi \Delta(\xi)=0
$$

or as

$$
\frac{\mathrm{d}^{2} \chi(\eta)}{\mathrm{d} \eta^{2}}-i \eta \chi(\eta)=0
$$

Equation (28) has two elementary solutions $\mathrm{Ai}(\xi)$ and $\mathrm{Ai}\left(\xi \mathrm{e}^{2 \pi i / 3}\right)$, where $\mathrm{Ai}$ are the well-known Airy functions (Abramowitz and Stegun, 1965). In terms of $\eta$ these elementary solutions are $\mathrm{Ai}\left(\eta \mathrm{e}^{\pi i / 6}\right)$ and $\mathrm{Ai}\left(\eta \mathrm{e}^{5 \pi i / 6}\right)$, which are solutions of Eqn. (29). This last equation is the starting equation 
of the analysis of Tollmien and Holstein. In terms of the solutions $\phi_{3}$ and $\phi_{4}$ of Eqn. (26), we find

$$
\frac{\mathrm{d}^{2} \phi_{3}}{\mathrm{~d} \eta^{2}}=c_{1} \operatorname{Ai}\left(\eta \mathrm{e}^{\pi i / 6}\right) \text { and } \frac{\mathrm{d}^{2} \phi_{4}}{\mathrm{~d} \eta^{2}}=c_{2} \operatorname{Ai}\left(-\eta \mathrm{e}^{-\pi i / 6}\right)
$$

where $c_{1}$ and $c_{2}$ are arbitrary constants and $\eta=z / \epsilon$. An important property of the Airy functions is that $\operatorname{Ai}(\bar{\xi})=\overline{\operatorname{Ai}(\xi)}$, where the overbar denotes the complex conjugate. This property enables us to relate $\phi_{4}$ to $\phi_{3}$. By choosing $c_{1}=-2 i(12)^{1 / 6}$ and $c_{2}=2 i(12)^{1 / 6}$, the right-hand terms of Eqn. (30) may be expressed in terms of modified Hankel functions (Drazin and Reid, 1982):

$h_{1}(i \eta)=-2 i(12)^{1 / 6} \operatorname{Ai}\left(\eta \mathrm{e}^{\pi i / 6}\right), \quad h_{2}(-i \eta)=2 i(12)^{1 / 6} \operatorname{Ai}\left(\eta \mathrm{e}^{-\pi i / 6}\right)$

$h_{1}(i \eta)$ together with its first and second integrals representing $\mathrm{d}^{2} \phi_{3} / \mathrm{d} \eta^{2}$, $\mathrm{d} \phi_{3} / \mathrm{d} \eta$ and $\phi_{3}$, respectively, have been tabulated by Singh et al. (1963) for the range $\eta=-10(0.1)+10$. With $c_{1}=-2(3)^{1 / 2} \mathrm{e}^{\pi i / 4}$ and $c_{2}=$ $2(3)^{1 / 2} \mathrm{e}^{\pi i / 4}$ we obtain expressions in terms of Hankel functions of the first and second kind of fractional order one-third. In the notation of Tollmien and Holstein, we have

$$
\begin{aligned}
& F_{1}(i \eta)=\eta^{1 / 2} H_{1 / 3}^{(1)}\left[\frac{2}{3}(i \eta)^{3 / 2}\right]=-2(3)^{1 / 2} \mathrm{e}^{\pi i / 4} \operatorname{Ai}\left(\eta \mathrm{e}^{\pi i / 6}\right) \\
& F_{2}(-i \eta)=(-\eta)^{1 / 2} H_{1 / 3}^{(2)}\left[\frac{2}{3}(-i \eta)^{3 / 2}\right]=2(3)^{1 / 2} \mathrm{e}^{\pi i / 4} \operatorname{Ai}\left(\eta \mathrm{e}^{-\pi i / 6}\right)
\end{aligned}
$$

Holstein (1950) tabulated $F_{1}$ and its first and second integrals for the range $\eta=-8(0.5)+8$. Further, Eqns. (31) and (32) show that $h_{2}(-i \eta)=h_{1}(i \eta)$ and $F_{2}(-i \eta)=-i F_{1}(i \eta)$. These relationships enable us to express $\mathrm{d}^{2} \phi_{4} / \mathrm{d} \eta^{2}, \mathrm{~d} \phi_{4} / \mathrm{d} \eta$ and $\phi_{4}$ in terms of $\mathrm{d}^{2} \phi_{3} / \mathrm{d} \eta^{2}, \mathrm{~d} \phi_{3} / \mathrm{d} \eta$ and $\phi_{3}$, respectively.

An approximate solution of Eqn. (5) satisfying (6) and (10) is now constructed in the following way. We start the numerical solution of Eqn. (20) at the free surface with initial conditions (21). At a certain level $z=z_{B}$ we continue this numerical solution with a linear combination

$Q(z)=A \phi_{1}(z)+B \phi_{2}(z)+C \phi_{3}(z)+D \phi_{4}(z)$

of the above-defined approximate expressions for the linear independent solution of Eqn. (5). The four complex-valued coefficients $A, B, C$ and $D$ are now determined in such a way that $Q$ and $Q^{\prime}$ are continuous at $z=z_{B}$ 
and satisfy the zero-velocity conditions (6) at the bottom, leading to

$$
\begin{aligned}
& A \phi_{1}\left(z_{B}\right)+B \phi_{2}\left(z_{B}\right)+C \phi_{3}\left(z_{B}\right)+D \phi_{4}\left(z_{B}\right)=Q\left(z_{B}\right) \\
& A \phi_{1}^{\prime}\left(z_{B}\right)+B \phi_{2}^{\prime}\left(z_{B}\right)+C \phi_{3}^{\prime}\left(z_{B}\right)+D \phi_{4}^{\prime}\left(z_{B}\right)=Q^{\prime}\left(z_{B}\right) \\
& A \phi_{1}(0)+B \phi_{2}(0)+C \phi_{3}(0)+D \phi_{4}(0)=0 \\
& A \phi_{1}^{\prime}(0)+B \phi_{2}^{\prime}(0)+C \phi_{3}^{\prime}(0)+D \phi_{4}^{\prime}(0)=0
\end{aligned}
$$

As the numerical solution of Eqn. (20) and the functions $\phi_{1}, \phi_{2}, \phi_{3}$ and $\phi_{4}$ are different approximations of the solution of Eqn. (5), we will find that only $Q$ and $Q^{\prime}$ are continuous in $z=z_{B}$. However, by a suitable choice of $z_{B}$, we may achieve also the result that $Q^{\prime \prime}$ is continuous in $z=z_{B}$ or has the smallest possible discontinuity.

It may be further noted here that the functions $\phi_{2}, \phi_{3}$ and $\phi_{4}$ are complex valued, indicating, according to Eqn. (4), that the streamfunction may have a wavy character also in the vertical direction. This corresponds to the fact that Eqn. (20) has a harmonic solution when $\left[\left(U^{\prime \prime} / U\right)+k^{2}\right]<0$, which will occur in a layer close to the bottom, as there $U^{\prime \prime}$ has large negative values. With increasing distance from the bottom the factor $\left[\left(U^{\prime \prime} / U\right)+k^{2}\right]$ will become positive and the solution has a real negative exponential character. For a thorough discussion of the behaviour of the solution of equations of the type of Eqn. (20) above and below a 'caustic layer', where $\left[\left(U^{\prime \prime} / U\right)+k^{2}\right]=0$, the reader is referred to Lighthill (1978). However, in the examples discussed in the next section $z_{B}$ may be chosen in the region where $\left[\left(U^{\prime \prime} / U\right)+k^{2}\right]>0$, as $U$ is the logarithmic velocity distribution and $k$ is chosen not too small. Then, according to Eqn. (4), the terms $Q\left(z_{B}\right)$ and $Q^{\prime}\left(z_{B}\right)$ on the right-hand side of Eqn. (34) simply represent the amplitudes of the oscillatory functions $\psi$ and $\mathrm{d} \psi / \mathrm{d} z$, respectively, at the level $z=z_{B}$ above the bottom.

Finally, the resulting approximate solution of Eqn. (5) satisfying the conditions (6) and (10) is normalized by imposing condition (19). As $\eta=z / \epsilon$ and $\epsilon=\left(k \operatorname{Re} U_{0}^{\prime}\right)^{-1 / 3}$, this is realized by multiplying $Q$ by the factor

$$
\frac{\left[2 S_{\zeta \zeta}(k)\right]^{1 / 2}}{\mathrm{Fr}^{2}\left|U_{0}^{\prime}\right| \cdot\left|\mathrm{d}^{3} Q / \mathrm{d} \eta^{3}\right|_{\eta=0}}
$$

At the bottom, the solution has the form $A \phi_{1}+B \phi_{2}+C \phi_{3}+D \phi_{4}$. Consequently, in view of Eqns. (22) and (24),

$$
\frac{\mathrm{d}^{3} Q}{\mathrm{~d} \eta^{3}}=B \epsilon \frac{U_{0}^{\prime \prime}}{U_{0}^{\prime}} \frac{\mathrm{d}^{3} \tilde{\phi}_{21}}{\mathrm{~d} \eta^{3}}+C \frac{\mathrm{d}^{3} \phi_{3}}{\mathrm{~d} \eta^{3}}+D \frac{\mathrm{d}^{3} \phi_{4}}{\mathrm{~d} \eta^{3}}, \quad \eta=0
$$


in which $\left(\mathrm{d}^{3} \tilde{\phi}_{21} / \mathrm{d} \eta^{3}\right)_{\eta=0}=0.938893$ (Holstein, 1950). Further, from Eqn. (30) we have

$$
\left(\frac{\mathrm{d}^{3} \phi_{3}}{\mathrm{~d} \eta^{3}}\right)_{\eta=0}=c_{1} \mathrm{e}^{\pi i / 6} \mathrm{Ai}^{\prime}(0), \quad\left(\frac{\mathrm{d}^{3} \phi_{4}}{\mathrm{~d} \eta^{3}}\right)_{\eta=0}=-c_{2} \mathrm{e}^{-\pi i / 6} \mathrm{Ai}^{\prime}(0)
$$

where $\mathrm{Ai}^{\prime}(0)=-0.25881940$ (Abramowitz and Stegun, 1965).

\section{NUMERICAL RESULTS}

The mean square values $\overline{u^{2}}$ and $\overline{w^{2}}$ of the horizontal and vertical disturbed velocities are calculated for various values of the flow and spectral parameters. In addition, the vertical derivative $\partial \overline{w^{2}} / \partial z$ is calculated. As we will see below, the behaviour of this quantity as a function of $z$ will lead to interesting conclusions. All these quantities are calculated as functions of the depth coordinate $z$ and are made dimensionless with the mean velocity $\bar{U}$ and the depth $d$ of the channel. Using Eqns. (3) and (4) we obtain for a single harmonic component with wavenumber $k$

$\overline{u^{2}}=\frac{1}{2} Q^{\prime 2}, \quad \overline{w^{2}}=\frac{1}{2} k^{2} Q^{2}, \quad \frac{\partial \overline{w^{2}}}{\partial z}=k^{2} Q Q^{\prime}$

We now substitute for $Q$ and $Q^{\prime}$ the solutions as obtained in the preceding section. We then obtain for the fluctuations owing to the total spectrum:

$\overline{u^{2}}=\frac{1}{2} \int_{0}^{\infty} Q^{\prime 2} \mathrm{~d} k, \quad \overline{w^{2}}=\frac{1}{2} \int_{0}^{\infty} k^{2} Q^{2} \mathrm{~d} k, \quad \frac{\partial \overline{w^{2}}}{\partial z}=\int_{0}^{\infty} k^{2} Q Q^{\prime} \mathrm{d} k$

It is noted that $\overline{u^{2}}, \overline{w^{2}}$ and $\partial \overline{w^{2}} / \partial z$ are continuous functions of the depth coordinate $z$, as $Q(z)$ and $Q^{\prime}(z)$ are continuous functions. For the vertical mean velocity distribution $U(z)$, we use the dimensionless expression

$U(z)=(1 / A)\left[\ln \left(z / z_{0}\right)+2.04\right]$

where $A=\int_{z_{0}}^{1} \ln \left(z / z_{0}\right) \mathrm{d} z+2.04$ is a constant, normalizing the mean velocity approximately to one, as $z_{0} \ll 1$. This expression is directly derived from the 'law of the wall' velocity profile (Coles, 1956)

$U / U_{\tau}=(1 / \kappa) \ln \left(z U_{\tau} / \nu\right)+C$

in which $U_{\tau}$ represents the friction velocity and $\nu\left(=10^{-6} \mathrm{~m}^{2} \mathrm{~s}^{-1}\right)$ is the kinematic viscosity. Coles defined $\kappa=0.40$ and $C=5.1$, leading to the constant 2.04 in Eqn. (40). Equation (41) is well established for $z U_{\tau} / \nu$ greater than about 50 . This leaves the shape of the profile very close to the bottom undetermined. For that reason we use Eqn. (40) in our calculations for $z_{0} \leqslant z \leqslant 1$ and assume a linear shape of the profile for $0 \leqslant z \leqslant z_{0}$. As another consequence, we have no well-determined values for the deriva- 
tives $U^{\prime}(0)$ and $U^{\prime \prime}(0)$ of the mean velocity at the bottom. These quantities appear as parameters in the approximate solutions of the OS equation defined in the preceding section. Further, in the calculations presented here the mean velocity profile and the spectral densities of the bottom waves have to be specified. For the spectral distribution function we use here the rather arbitrary shape

$$
\begin{aligned}
S_{\zeta \zeta}(k) & =C \exp \left[\left(k-k_{m}\right)^{2} / 2 \sigma^{2}\right] & & k_{b} \leqslant k \leqslant k_{e} \\
& =0, & & \text { elsewhere }
\end{aligned}
$$

where we assume in our calculations $k_{m}=\left(k_{b}+k_{e}\right) / 2, \sigma=0.5$ and $C=$ 0.001465 . This value for $C$ corresponds to a spectral area equal to 0.001 and a significant wave height (Ochi, 1982) equal to 0.12681. It is noted that, in view of Eqn. (19), the values of $\overline{u^{2}}$ and $\overline{w^{2}}$ are proportional to the total surface of the bed wave spectrum.

Resuming, we have as parameters related to the mean velocity: $z_{0}$ (which we fix at the value 0.001 ), the depth $d$ and the mean velocity $\bar{U}$, or the Froude number Fr and the Reynolds number Re, and finally, the parameters $U^{\prime}(0)$ and $U^{\prime \prime}(0)$ characterizing the mean velocity profile in the boundary layer. As spectral density parameters we have only the boundaries $k_{b}$ and $k_{e}$, as $k_{m}, \sigma$ and $C$ are given fixed values or have been expressed in $k_{b}$ and $k_{e}$.

In Figs. 1-3 we give results for the r.m.s. values $\left(\overline{u^{2}}\right)^{1 / 2}$ and $\left(\overline{w^{2}}\right)^{1 / 2}$ of the horizontal and vertical velocity disturbances and for the gradient $\overline{\partial w^{2}} / \partial z$. In Fig. 1 results are given for various values of $U^{\prime}(0)$ and $U^{\prime \prime}(0)$. In Fig. 2 only the spectral interval is varied, and in Fig. 3 only the mean velocity in the channel is varied. The spectrum $S_{\zeta \zeta}(k)$ has been discretized with respect to $k$ with a step length equal to 0.0125 . For each wavenumber the approximate solution is determined as described in the preceding section; this includes the determination of a point $z=z_{B}$, where the numerical solution of Eqns. (21) and (22) is continued with a linear combination (33). As $z_{B}$ is fixed at a point where $Q^{\prime \prime}$ is also continuous or has the smallest possible jump, this point generally differs for the various values of $k$. The range of these points $z_{B}$ for the values of $k$ in the spectrum is between the plus signs on the curves. Figure 1 shows clearly the sensitivity of the results for variation in the values of $U^{\prime}(0)$ and $U^{\prime \prime}(0)$. Obviously, a rather accurate description of the velocity profile close to the bottom is desirable. Unfortunately, this is not possible for the velocity profiles used in engineering practice, in particular above a rough bottom. A striking feature of Fig. 1 is that also in a layer at the free surface, velocity disturbances are generated by the bottom irregularities. This is because the flow may have stationary surface waves for a wavenumber in the range $6<k<7$. De Jong (1989) showed that these so-called free waves of the flow are approximately 
(a)
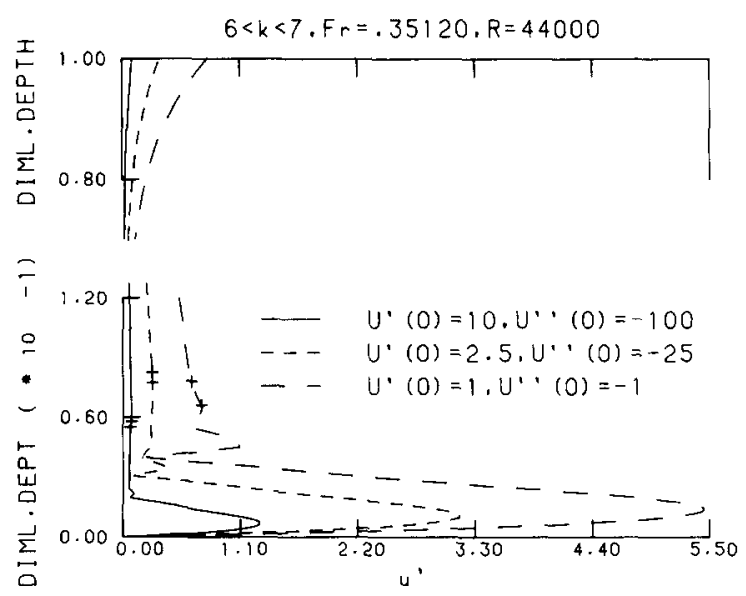

(b)

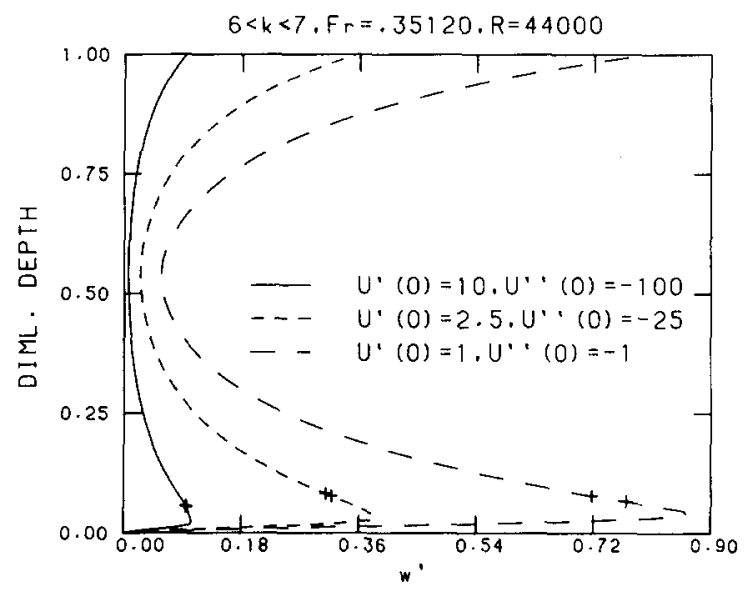

(c)
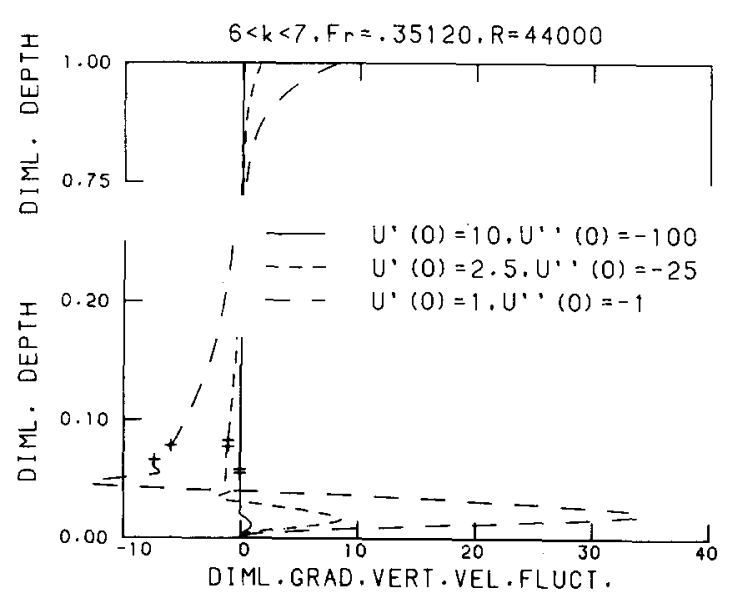

Fig. 1. (a) $u^{\prime}=\left(\overline{u^{2}}\right)^{1 / 2} / \bar{U}$ of horizontal velocity fluctuations; (b) $w^{\prime}=\left(\overline{w^{2}}\right)^{1 / 2} / \bar{U}$ of vertical velocity fluctuations; (c) gradient $\left(\partial \overline{w^{2}} / \partial z\right) d / \bar{U}^{2}$ of vertical velocity fluctuations; for various values of $U^{\prime}(0)$ and $U^{\prime \prime}(0)$. Viscous and inviscid solutions are connected in the range between the + signs. 
obtained by solving Eqns. (20) and (21) over the whole flow depth, $0 \leqslant z \leqslant 1$. The wavenumber of the free waves is now the value of $k$ that gives a zero value for $Q$ at the bottom. For the flow conditions of Fig. 1 the free wave has the wavenumber $k=6.40$, which is in the range $6<k<7$ of the spectrum. This fact is also observed in Fig. 3 for the case $\bar{U}=0.35, D=0.1$, which has the eigenvalue $k=6.33$. For the other values of $\bar{U}$ in Fig. 3, and also for the flow conditions in Fig. 2, the eigenvalues are outside the spectral range of the bottom irregularities. The disturbances at the free surface are then not observed or occur only very slightly. De Jong (1989) showed that for Froude numbers up to about 0.5-0.6 these eigenvalues satisfy approximately the relation

$c_{f}=\frac{1}{\operatorname{Fr}}\left(\frac{1}{k} \tanh k\right)^{1 / 2}$

which is the dispersion relationship of potential flow (Stoker, 1957). $c_{f}$ is the celerity of the free surface waves, which has for the eigenvalue $k$ a value equal to the fluid velocity at the free surface. The free surface waves are then stationary when they propagate in a direction opposite to the direction of the fluid flow.

In this paper we have considered two-dimensional velocity fluctuations which may have a dominant character only in certain special situations of resonance. Experimental data are related in general to fully developed turbulence which has essentially a three-dimensional character. Consequently, only a qualitative comparison is possible in terms of observed trends. For this comparison we will choose the wind tunnel data of Corrsin and Kistler (1954) and the water tunnel data of Sleath (1987). Although these flows are of a different type, the results presented in Figs. 1(a), 1(b), 2(a) and 2(b) show trends very similar to those observed in these measurements: The r.m.s. values $\left(\overline{u^{2}}\right)^{1 / 2}$ and $\left(\overline{w^{2}}\right)^{1 / 2}$ of the horizontal and vertical velocity fluctuations rise to a maximum and then decline with increasing distance from the bottom. The maximum of $\left(\overline{u^{2}}\right)^{1 / 2}$ occurs closer to the bed than the maximum of $\left(\overline{w^{2}}\right)^{1 / 2}$. The maximum value of $\left(\overline{u^{2}}\right)^{1 / 2}$ is also significantly larger than that for $\left(\overline{w^{2}}\right)^{1 / 2}$.

We conclude this section with some tentative conclusions from the variations of $\overline{\partial w^{2}} / \partial z$ as observed in Figs. 1(c), 2(c) and 3, when we vary the flow or spectral parameters in the channel. It is well known (Irmay, 1960) that $\partial \overline{w^{2}} / \partial z$ contributes to the mean vertical particle acceleration as a result of inhomogeneities of turbulence in the vertical direction. This is easy to see when we substitute in Eqn. (1) the three-dimensional velocity $(U+u, V+v, W+w)$, which is the sum of mean value $(U, V, W)$ and the disturbances $(u, v, w)$. Averaging and using the continuity equation yields 
(a)
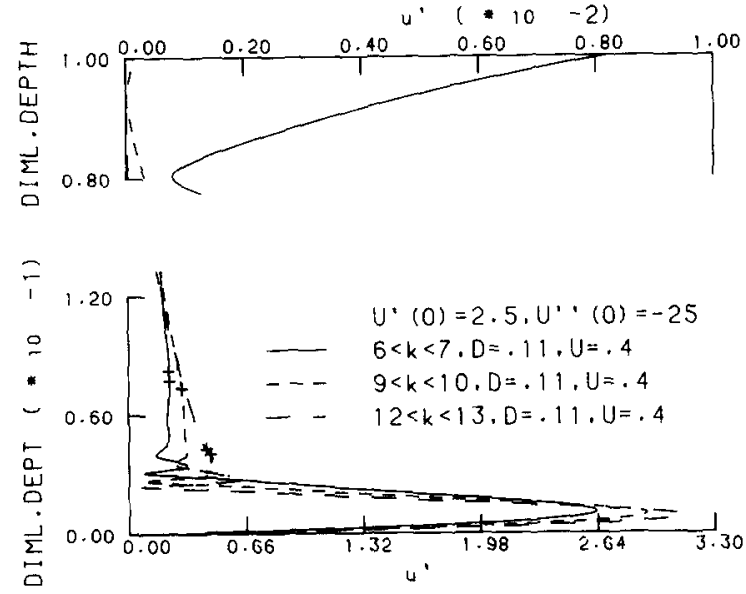

(b)

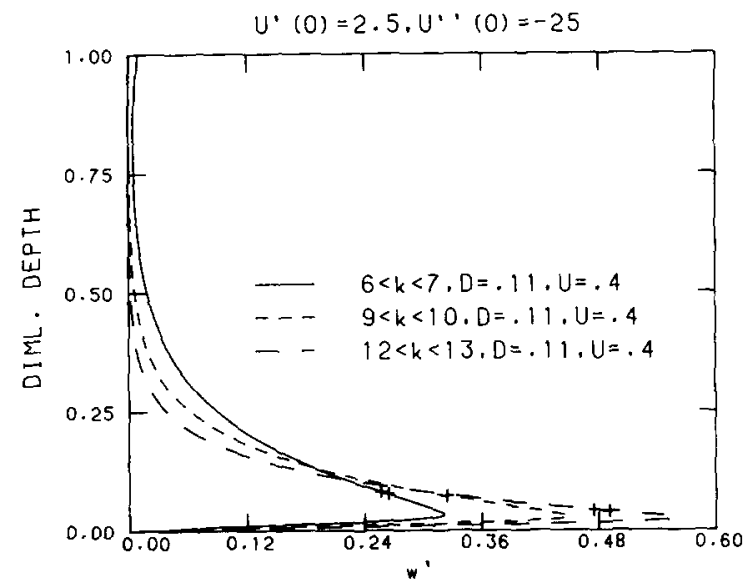

(c)
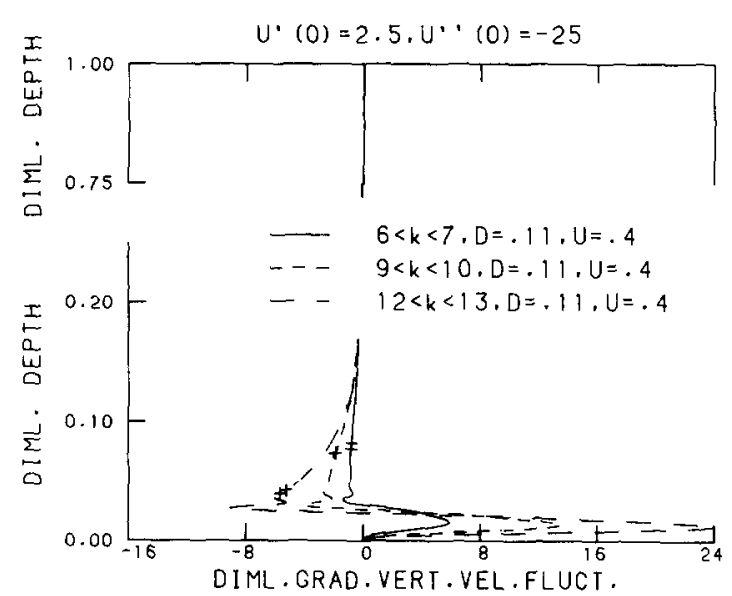

Fig. 2. (a) $\bar{u}^{\prime}=\left(\overline{u^{2}}\right)^{1 / 2} / \bar{U}$ of horizontal velocity fluctuations; (b) $w^{\prime}=\left(\overline{w^{2}}\right)^{1 / 2} / \bar{U}$ of vertical velocity fluctuations; (c) gradient $\left(\partial \bar{w}^{2} / \partial z\right) d / \bar{U}^{2}$ of vertical velocity fluctuations; for various wavenumber ranges. Viscous and inviscid solutions are connected in the range between the + signs. 


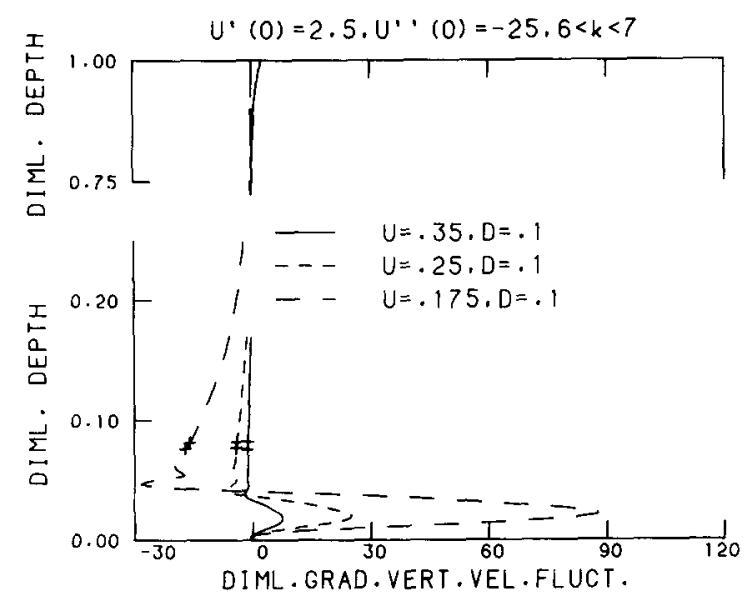

Fig. 3. Gradient $\left(\partial \overline{w^{2}} / \partial z\right) d / \bar{U}^{2}$ of vertical velocity fluctuations for various mean velocities in the channel. Viscous and inviscid solutions are connected in the range between the + signs.

the following expression for the vertical acceleration of a fluid particle:

$$
\begin{aligned}
\frac{\mathrm{d} W}{\mathrm{~d} t} & =\frac{\partial W}{\partial t}+\frac{\partial U W}{\partial x}+\frac{\partial V W}{\partial y}+\frac{\partial W^{2}}{\partial z}+\frac{\partial \overline{u w}}{\partial x}+\frac{\partial \overline{v w}}{\partial y}+\frac{\partial \overline{w^{2}}}{\partial z} \\
& =-g+\nu\left(\frac{\partial^{2} W}{\partial x^{2}}+\frac{\partial^{2} W}{\partial y^{2}}+\frac{\partial^{2} W}{\partial z^{2}}\right)
\end{aligned}
$$

For the velocity fluctuations generated by one-dimensional longitudinal irregular bed waves only the Reynolds stress $\partial \overline{w^{2}} / \partial z$ in Eqn. (44) is retained. For that reason, variations of $\partial \overline{w^{2}} / \partial z$ in the flow field may contribute to the generation of secondary flows. Such variations may be caused in a channel by, for example, lateral variations in depth and velocity. For the same reason we may expect that secondary flows are also generated in flow systems where we have regions with different bottom roughness. Further, Figs. 1(c), 2(c) and 3 show that $\partial \overline{w^{2}} / \partial z$ changes sign one or more times in the vertical direction. This suggests that secondary flows may occur as one or more recirculating cells one above the other. This corresponds, in a qualitative sense, to the measurements in a rectangular channel of Melling and Whitelaw (1977), who observed the existence of these cells.

\section{SUMMARY AND DISCUSSION}

In a one-dimensional channel flow the velocity fluctuations generated by one-dimensional irregular bottom waves are calculated. The velocity fluctuations are supposed to have a two-dimensional character and to be stable 
and stationary. Therefore, phenomena such as bursting, breaking of waves and other non-stationary phenomena are not included. The rough bottom is characterized by its spectral density function, and the flow is characterized by the mean velocity profile, the Froude number and the Reynolds number. The velocity fluctuations are supposed to be governed by the Orr-Sommerfeld (OS) equation. This linear equation relates the amplitudes of the horizontal and vertical velocity fluctuations in the fluid to the amplitude of the harmonic bottom corrugation. The velocities generated by the irregular bottom are obtained by superposition. The OS equation is solved in an approximate manner. In the upper layer of the flow it is reduced to the Rayleigh equation. The numerical solution of this equation that satisfies the free surface condition is continued in the boundary layer by a linear combination of the elementary solutions of the OS equation. These solutions are determined in a heuristic way by reducing the OS equation to appropriate forms. One solution is a regular series solution of the Rayleigh equation. The second solution has been viscously corrected to avoid singular behaviour at the bottom but has at a large distance from the bottom the same inviscid behaviour as the other elementary solution of the Rayleigh equation. The other two elementary solutions of the OS equation are everywhere viscously dominated and are approximated by truncating the OS equation to an equation which has only the highest derivatives of the non-viscous terms. These four elementary solutions in the boundary layer are appropriately combined in such a way that the disturbed velocities and their derivatives are everywhere continuous; furthermore, the zerovelocity conditions at the bottom are satisfied. The mean velocity in the boundary layer above the irregular bottom is characterized by the derivatives $U^{\prime}(0)$ and $U^{\prime \prime}(0)$ of the mean velocity at the bottom.

Many simplifications and approximations have been made to formulate and solve the boundary value problem describing two-dimensional disturbed flow. For that reason it is clear that a comparison with experiments relating to three-dimensional fully developed turbulence can only be made in a qualitative sense. In view of this, it is a rather striking feature that the calculated r.m.s. values $\left(\overline{u^{2}}\right)^{1 / 2}$ and $\left(\bar{w}^{2}\right)^{1 / 2}$ of the horizontal and vertical velocity disturbances show a very similar trend to measurements in fully developed turbulence. Another interesting point is that the numerical results enable us to explain the generation of secondary flows in systems with varying flow and spectral parameters. Also, the cellular character of these flows can be explained.

\section{REFERENCES}

Abramowitz, M. and Stegun, I.A. (Editors), 1965. Handbook of Mathematical Functions, Dover, New York, p. 446. 
Betchov, R. and Criminale, Jr., W.O., 1967. Stability of Parallel Flows. Academic Press, New York, pp. 74-96.

Coles, D., 1956. The law of the wake in the turbulent boundary level. J. Fluid Mech., 1: 191-226.

Corrsin, S. and Kistler, A.L., 1954. The free-stream boundaries of turbulent flows. NACA Tech. Note 3133 NACA Rep. 1244 (1955).

De Jong, B., 1989. Bed waves generated by internal waves in alluvial channels. J. Hydraul. Eng., 115 (6): 801-817.

Drazin, P.G. and Reid, W.H., 1982. Hydrodynamic Stability. Cambridge University Press, Cambridge, pp. 124-285.

Holstein, H., 1950. Über die äussere und innere Reibungsschicht bei Störungen Laminarer Strömungen, Z. Angew. Math. Mech., 30 (1/2): 25-49.

Irmay, S., 1960. Accelerations and mean trajectories in turbulent channel flow. J. Basic Eng., 82(D): 961-972.

Kranenburg, C., Pietrzak, J.D. and Abraham, G., 1991. Trapped internal waves over undular topography. J. Fluid Mech., 226: 205-217.

Landahl, M.T., 1967. A wave-guide model for turbulent shear flow. J. Fluid Mech., 29(3): 441-459.

Lighthill, J., 1978. Waves in Fluids, Cambridge University Press. Cambridge, pp. 284-436.

Melling, A. and Whitelaw, J.H., 1977. Turbulent flow in a rectangular duct. J. Fluid Mech., 78(2): 289-315.

Ochi, M.K., 1982. Stochastic analysis and probabilistic prediction of random seas. In: VenTe Chow (Editor), Advances in Hydroscience. Vol. 13, Academic Press, New York, pp. 218-369.

Pierson, W.J., Neumann, G. and James, R.W., 1958. Practical methods for observing and forecasting ocean waves by means of wave spectra and statistics. Hydrogr. Off. Publ. 603. US. Navy Hydrographic Office, Washington, DC, 284 pp.

Raudkivi, A.J., 1990. Loose Boundary Hydraulics, 3rd edn. Pergamon, New York, pp. $40-41$.

Reid, W.H., 1965. The stability of parallel flows. In: M. Holt (Editor), Basic Developments in Fluid Dynamics, Vol. 1. Academic Press, New York, pp. 249-307.

Rodi, W., 1980. Turbulence Models and their Application in Hydraulics. Int. Assoc. for Hydraul. Res., Delft, pp. 5-34.

Schlichting, H., 1968. Boundary-Layer Theory. McGraw-Hill, New York, pp. 121-123.

Singh, K., Lumley, J.K. and Betchov, R., 1963. Modified Hankel Functions and their integrals to argument 10. Pa. State Univ. Coll. Eng. Eng. Res. Bull., B-87, pp. 1-29.

Sleath, J.F., 1987. Turbulent oscillatory flows over rough beds. J. Fluid Mech., 182: 369-409.

Stoker, J.J., 1957. Water Waves. Interscience, New York, pp. 198-210.

Stuart, J.T., 1963. Hydrodynamic stability. In: L. Rosenhead (Editors), Laminar Boundary Layers. Oxford University Press, London, pp. 492-579.

Tollmien, W., 1929. Über die Entstehung der Turbulenz. 1. Mitteilung Nachr. Ges. Wiss. Göttingen, Math.-Phys. Kl., 21-44.

Tollmien, W., 1947. Asymptotische Integration der Störungsdifferentialgleichung ebener laminarer Strömungen bei hohen Reynoldsschen Zahlen. Z. Angew. Math. Mech., 25/27: 33-50, 70-83.

Turner, J.S., 1973. Buoyancy Effects in Fluids. Cambridge University Press, Cambridge, pp. $1-90$. 